\title{
Choreography of AMPK activation
}

\author{
Cell Research (2015) 25:5-6. doi:10.1038/cr.2014.163; published online 5 December 2014
}

\begin{abstract}
A recent study published in Cell Research by $\mathrm{Li}$ and colleagues reports a detailed biophysical and structural study of AMPK's intra-molecular interactions during activation. By employing subunit tagging and proximity analysis with the aid of AlphaScreen instrumentation, Li et al. add to our understanding of the choreography of activation of AMPK by both nucleotides and phosphorylation.
\end{abstract}

AMPK activation is triggered by energy depletion resulting in rises in cellular AMP. AMPK phosphorylates many proteins to switch off energyconsuming pathways and switch on energy-generating pathways. As many age-onset diseases involve metabolic disorders, there has been considerable interest in developing AMPK-activating drugs. Understanding the biochemistry and structure-function relationships of AMPK has been challenging due in part to its complex $\alpha \beta \gamma$ heterotrimeric structure, multiple ligand-binding sites and extensive posttranslational modifications. Each subunit of the heterotrimer has multiple alternate isoforms $(\alpha 1-2$, $\beta 1-2$ and $\gamma 1-3)$, with each isoform harboring multiple functional domains. Recent structural information has been a boon to AMPK researchers with two $(\alpha 2 \beta 1 \gamma 1$ and $\alpha 1 \beta 1 \gamma 1)$ near fulllength crystal structures of activated phosphorylated AMPK bound to AMP, with and without AMPK-activating drugs Merck 991 or A-769662 [1, 2]. The new findings reported by Li et al. [3] focus on an autoinhibitory domain (AID) in the catalytic $\alpha$-subunit and a carbohydrate-binding module (CBM) in the $\beta$-subunit $[4,5]$. In the basal state the
AID interacts with the kinase domain (KD), but is sequestered to the $\gamma$-subunit upon activation $[6,7]$. The phosphorylated CBM sits on the N-lobe of the $\alpha$-subunit $\mathrm{KD}$, forming what we now term the allosteric drug and metabolite (ADaM)-binding pocket. Despite this wealth of structural information, little is known about the inactive form of AMPK, including how nucleotides act to inhibit or activate AMPK.

ATP binds to the AMPK $\gamma$-subunit in the inactive conformation, maintaining the $\alpha$-catalytic subunit in its autoinhibited form via AID interactions. AMP binding to the $\gamma$-subunit displaces ATP and triggers AMPK activation. This event is sensed by the $\alpha$-subunit regulatory subunit-interacting motif $(\alpha$ RIM) and disengages the AID from the KD. Phosphorylation of the activation loop at $\alpha-\mathrm{T} 172 / \mathrm{T} 174$ by upstream kinases then locks AMPK in the active conformation.

To examine enzyme transitions from the inactive to the active state $\mathrm{Li}$ and colleagues have used tags attached at differing $\mathrm{N}$ - and/or C-termini throughout the AMPK heterotrimer, which bind to donor and acceptor beads [3]. The AlphaScreen luminescence proximity assay can sense the proximity of one domain to another, and in this way the change in proximity between two domains can be assessed under different nucleotide-binding conditions. AMP causes the $\mathrm{N}$-terminal regions of the $\alpha$-subunit and $\gamma$-subunit to move closer together $\left(\mathrm{p}^{\mathrm{AMP}}\right)$, whereas ATP causes these two regions to dissociate ( $\mathrm{p}^{\mathrm{ATP}}$ ) (Figure 1A and 1B). By comparison, the addition of AMP or ATP does not change the proximity of the $\alpha-\beta$ and $\beta-\gamma$ subunit interactions. Thus, the inactive AMPK (ATP-bound) has an extended conformation, while the AMP-bound form is more compact, in agreement with conclusions drawn from earlier SAXS studies [8].

Li et al. solved the X-ray crystal structure of the previously recalcitrant human KD-AID enzyme fragment by introducing the entropy reduction mutation K43A [1]. The human KD-AID structure showed that the AID motif bound to the KD hinge region, holding $\mathrm{KD}$ in an inactive open conformation (Figure 1A). This observation was confirmed by a series of mutations, which abolished the inhibitory effect of the AID on the KD. These mutations in fulllength AMPK also eliminate the AMP regulation of kinase activity, strongly supporting previous results [4].

Remarkably the nucleotide regulation of AMPK by AMP and ATP could be recapitulated with fragments of the enzyme including the AMPK core complex ( $\alpha 1$ [395-550]- $\beta 2$ [187-272]- $\gamma 1)$ and an AID- $\alpha$ RIM fragment ( $\alpha 1$ [282392]). Upon AMP binding to the core complex, the proximal distance of the AID- $\alpha$ RIM motif to the core decreased whereas upon ATP binding the proximal distance increased. These results were supported by previously reported mutations that cause AMPK to become AMP-independent, which inhibited the core complex from interacting with the AID [7].

The CBM of the $\beta$-subunit (Figure 1C), which is critical for the AMPK allosteric activation by several drugs [2], is known to bind glycogen and cyclodextran (CD). However, a physiological role for the glycogen interac- 


$$
\begin{aligned}
& \text { A ATP bound } \\
& \text { (inactive) }
\end{aligned}
$$

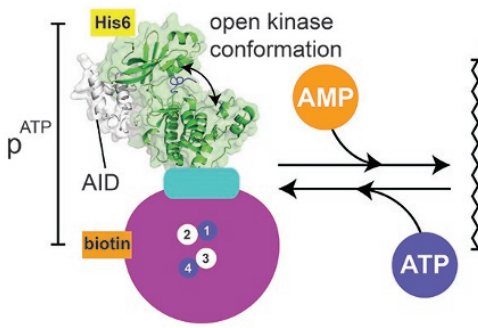

\section{B AMP bound (Pseudo-active)}

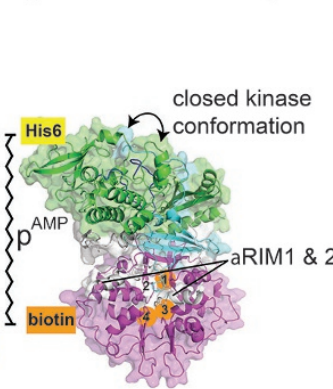

\section{AMP and CD bound (active)}

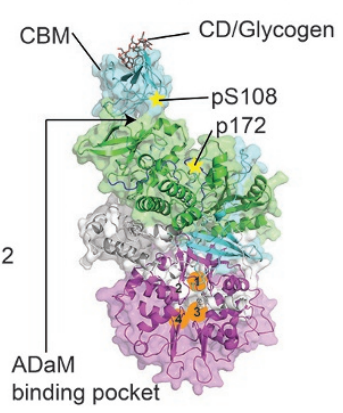

Figure 1 Nucleotide inhibition and activation of AMPK. Cartoon and surface representations of nucleotide-bound AMPK X-ray crystal structures. $\alpha$-subunit (green) including the KD and the AID- $\alpha$ RIM (white); $\beta$-subunit (cyan) including the CBM; $\gamma$-subunit (magenta) with nucleotide-binding (CBS) sites 1-4 labeled. AlphaScreen tags on the $\mathrm{N}$-terminus of the $\alpha$-subunit (His) and $\gamma$-subunit (biotin) are shown. (A) Inactive KD bound to the AID, with an animated $\gamma$-subunit showing CBS sites 1 and 4 occupied by ATP (blue), and 2 and 3 unbound. The AID can be seen bound to the hinge region of the $K D$, maintaining an inactive open conformation. (B) Unphosphorylated AMPK with AMP bound to $\gamma$-subunit CBS sites 1, 3 and 4. The heterotrimer has adopted a compact conformation with the proximity between the two AlphaScreen tags $\left(p^{\text {AMP }}\right)$ closer than that of ATP-bound AMPK $\left(p^{\text {ATP }}\right)$. The KD has adopted a closed pseudo-active conformation. (C) AMPK phosphorylated on $\alpha$ T172 (KD) and $\beta S 108$ (CBM), with AMP bound to the $\gamma$-subunit CBS sites 1, 3 and 4. The CBM is bound to the N-lobe of the KD, forming the ADaM-binding pocket, with $C D$ bound to the opposite face of the CBM.

tion has not been conclusively shown. Li et al. reveal that the interaction between isolated CBM and KD can be reduced upon glycogen or $\mathrm{CD}$ binding to the CBM [3]. Blocking CD/glycogen binding by mutating residue W99 in the CBM partially reversed the reduction in CBM-KD interaction caused by glycogen/CD binding. As originally reported by Polekhina et al. [9], Li et al. found that glycogen had no effect on AMPK activity and failed to reproduce the claims by Hardie and colleagues that glycogen inhibits AMPK activity [10].

As stated earlier, AMPK is an im- portant drug target. While some drugs show $\beta$-isoform selectivity, others can activate either $\beta 1$ or $\beta 2$ isoforms. While previous structures have revealed the drug-binding pocket of $\beta 1$-containing AMPK [1, 2], this report by Li et al. [3] represents the first time that the $\beta 2$ allosteric binding pocket has been visualized. Interestingly, the allosteric binding pockets of the two isoforms superimpose closely, suggesting a shared mechanism. The ADaM-binding pocket was formed in the absence of any allosteric activating drugs, such as Merck 991 or A-769662. This is in agreement with a recently reported rat $\alpha 1 \beta 1 \gamma 1$ structure [2].

Collectively, the study by $\mathrm{Li}$ and colleagues extends our understanding of how AMPK is physiologically activated and inactivated. This study demonstrated that the AID- $\alpha$ RIM element is responsible for sustaining either the active or inactive state as had previously been inferred from structural studies [ 1 , $2,4,6,11]$.

\section{Christopher G Langendorf ${ }^{1}$, Bruce E Kemp ${ }^{1}$}

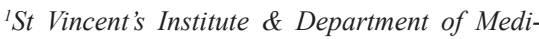
cine, The University of Melbourne, 41 Victoria Parade, Fitzroy, Victoria 3065, Australia Correspondence: Bruce E Kemp

E-mail: bkemp@svi.edu.au

\section{References}

1 Xiao B, Sanders MJ, Carmena D, et al. Nat Commun 2013; 4:3017.

2 Calabrese MF, Rajamohan F, Harris MS, et al. Structure 2014; 22:1161-1172.

3 Li X, Wang L, Zhou XE, et al. Cell Res 2015; 25:50-66.

4 Chen L, Jiao ZH, Zheng LS, et al. Nature 2009; 459:1146-1149.

5 Amodeo GA, Rudolph MJ, Tong L. Nature 2007; 449:492-495.

6 Xiao B, Sanders MJ, Underwood E, et al. Nature 2011; 472:230-233.

7 Chen L, Wang J, Zhang YY, et al. Nat Struct Mol Biol 2012; 19:716-718.

8 Riek U, Scholz R, Konarev P, et al. J Biol Chem 2008; 283:18331-18343.

9 Polekhina G, Gupta A, Michell BJ, et al. Curr Biol 2003; 13:867-871.

10 McBride A, Ghilagaber S, Nikolaev A, et al. Cell Metab 2009; 9:23-34.

11 Chen L, Xin FJ, Wang J, et al. Nature 2013; 498:E8-E10. 